\title{
Towards reference values of pericoronary adipose tissue attenuation: impact of coronary artery and tube voltage in coronary computed tomography angiography
}

\author{
Runlei Ma ${ }^{1,2} \cdot$ Daan Ties $^{3} \cdot$ Marly van Assen ${ }^{1} \cdot$ Gert Jan Pelgrim $^{1} \cdot$ Grigory Sidorenkov $^{4} \cdot$ Peter M. A. van Ooijen ${ }^{5,6}$. \\ Pim van der Harst ${ }^{3} \cdot$ Randy van Dijk $^{3} \cdot$ Rozemarijn Vliegenthart ${ }^{1}$
}

Received: 26 March 2020 / Revised: 28 May 2020 / Accepted: 3 July 2020 / Published online: 22 July 2020

(C) The Author(s) 2020

\begin{abstract}
Objectives To determine normal pericoronary adipose tissue mean attenuation ( $\left.\mathrm{PCAT}_{\mathrm{MA}}\right)$ values for left the anterior descending (LAD), left circumflex (LCX), and right coronary artery (RCA) in patients without plaques on coronary CT angiography (cCTA), taking into account tube voltage influence.

Methods This retrospective study included 192 patients (76 (39.6\%) men; median age 49 years (range, 19-79)) who underwent cCTA with third-generation dual-source CT for the suspicion of CAD between 2015 and 2017. We selected patients without

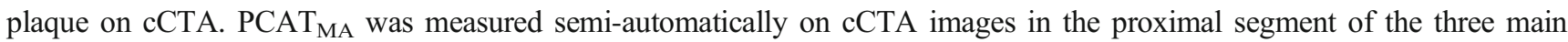
coronary arteries with $10 \mathrm{~mm}$ length. Paired $t$-testing was used to compare PCAT $\mathrm{MA}_{\mathrm{MA}}$ between combinations of two coronary

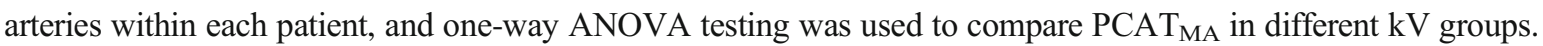

Results The overall mean \pm standard deviation (SD) PCAT $_{\mathrm{MA}}$ was $-90.3 \pm 11.1 \mathrm{HU}$ PCAT $_{\mathrm{MA}}$ in men was higher than that in women: $-88.5 \pm 10.5 \mathrm{HU}$ versus $-91.5 \pm 11.3 \mathrm{HU}(p=0.001)$. PCAT $_{\mathrm{MA}}$ of LAD, LCX, and RCA was $-92.4 \pm 11.6 \mathrm{HU},-88.4 \pm$ 9.9 HU, and $-90.2 \pm 11.4 \mathrm{HU}$, respectively. Pairwise comparison of the arteries showed significant difference in PCAT $\mathrm{MA}_{\mathrm{MA}}$. $\mathrm{LAn}$ LCX $(p<0.001), \mathrm{LAD}$ and RCA $(p=0.009), \mathrm{LCX}$ and RCA $(p=0.033) . \mathrm{PCAT}_{\mathrm{MA}}$ of the $70 \mathrm{kV}, 80 \mathrm{kV}, 90 \mathrm{kV}, 100 \mathrm{kV}$, and $120 \mathrm{kV}$ groups was $-95.6 \pm 9.6 \mathrm{HU},-90.2 \pm 11.5 \mathrm{HU},-87.3 \pm 9.9 \mathrm{HU},-82.7 \pm 6.2 \mathrm{HU}$, and $-79.3 \pm 6.8 \mathrm{HU}$, respectively $(p<0.001)$.

Conclusions In patients without plaque on cCTA, PCAT $_{M A}$ varied by tube voltage, with minor differences in PCAT MA $_{\text {between }}$

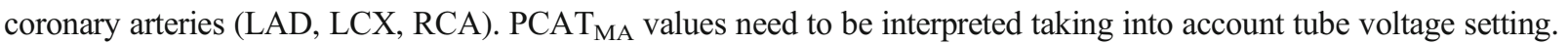

Key Points

- In patients without plaque on cCTA, PCAT $T_{M A}$ differs slightly by coronary artery (LAD, LCX, RCA).

- Tube voltage of cCTA affects PCAT $T_{M A}$ measurement, with mean PCAT $T_{M A}$ increasing linearly with increasing $\mathrm{kV}$.

- For longitudinal cCTA analysis of PCAT $T_{M A}$, the use of equal $k V$ setting is strongly recommended.
\end{abstract}

Keywords Computed tomography angiography · Adipose tissue $\cdot$ Coronary vessels/diagnostic imaging $\cdot$ Reproducibility of results $\cdot$ Atherosclerosis

Electronic supplementary material The online version of this article (https://doi.org/10.1007/s00330-020-07069-0) contains supplementary material, which is available to authorized users.

Rozemarijn Vliegenthart

r.vliegenthart@umcg.nl

1 Department of Radiology, University of Groningen, University Medical Center Groningen, Hanzeplein 1, 9713, GZ Groningen, the Netherlands

2 Department of Radiology, Affiliated Hospital of Nanjing University of Chinese Medicine, Nanjing, China
3 Department of Cardiology, University of Groningen, University Medical Center Groningen, Groningen, the Netherlands

4 Department of Epidemiology, University of Groningen, University Medical Center Groningen, Groningen, the Netherlands

5 Department of Radiation Oncology, University of Groningen, University Medical Center Groningen, Groningen, the Netherlands

6 Data Science Center in Health, University of Groningen, University Medical Center Groningen, Groningen, the Netherlands 


$\begin{array}{ll}\text { Abbreviations } \\ \text { BMI } & \text { Body mass index } \\ \text { CAD } & \text { Coronary artery disease } \\ \text { cCTA } & \text { Coronary computed tomography angiography } \\ \text { EAT } & \text { Epicardial adipose tissue } \\ \text { ECG } & \text { Electrocardiography } \\ \text { kV } & \text { Kilovoltage } \\ \text { LAD } & \text { Left anterior descending coronary artery } \\ \text { LCX } & \text { Left circumflex coronary artery } \\ \text { PCAT } & \text { Pericoronary adipose tissue mean attenuation } \\ \text { RCA } & \text { Right coronary artery } \\ \text { SD } & \text { Standard deviation }\end{array}$

\section{Introduction}

Coronary artery disease (CAD) is caused by atherosclerosis of the coronary arteries. Prior studies showed that coronary inflammation plays an essential role in the development and progression of atherosclerotic plaque [1-3]. An observational study demonstrated that invasively determined inflammatory changes of the coronary wall are present in early stages of CAD [4]. In the CANTOS trial, anti-inflammatory therapy reduced cardiovascular events, independent of lipid-lowering therapies [5, 6]. Efforts have been made to find a reliable noninvasive imaging parameter to detect coronary inflammation, focusing on adipose tissue [7-10]. The amount of epicardial adipose tissue (EAT) has been quantified [11-13], not only based on coronary computed tomography angiography (cCTA) but also based on coronary calcium scans or nongated chest $\mathrm{CTs}[14,15]$. More recently, attention was focused on pericoronary adipose tissue (PCAT). Although PCAT is part of EAT, morphological and functional characteristics of PCAT are different from those of EAT. PCAT is directly affected by coronary inflammation, causing compositional changes of PCAT, while EAT is mainly affected by systemic conditions such as obesity [16]. A clinical pathology review suggested PCAT to be an independent risk factor for cardiovascular disease [17]. Antonopoulos et al indirectly evaluated coronary inflammation on cCTA around the RCA by measuring the fat attenuation index, equivalent to PCAT mean attenuation $\left(\mathrm{PCAT}_{\mathrm{MA}}\right)$ [16]. They found a correlation between cCTA-derived PCAT $_{\mathrm{MA}}$ and adipocyte size or PCAT lipid volume in ex vivo PCAT histology. Additionally, PCAT and the coronary wall had a bidirectional communication, where inflammatory processes in the coronary vessel wall influenced PCAT composition via a paracrine pathway [16, 18]. In turn, PCAT influenced the coronary wall by secreted bioactive inflammation molecules [19]. In the presence of increased inflammation, higher CT attenuation of PCAT is expected [16].

Thus, PCAT could potentially be used as a non-invasive proxy to assess coronary inflammation based on routine
cCTA imaging, and could offer valuable information for early diagnosis, treatment, and prevention of CAD. Several studies explored the diagnostic value of $\mathrm{PCAT}_{\mathrm{MA}}$ in patients with plaques [20-24]. However, studies including patients without plaque so far mainly focused on the healthy RCA in small cohorts of patients. Further standardization and validation, as well as reference PCAT $\mathrm{MA}_{\mathrm{MA}}$ values in all three main coronary arteries without plaque, are needed before generalized clinical implementation can be considered. Reference values of PCAT $_{M A}$ for healthy patients are necessary for the application in diseased patients because based on the healthy reference values clinicians may in the future be able to classify the coronary arteries into healthy or vulnerable vessels, even before the presence of plaque. Additionally, clinical cCTA scans are acquired at different tube voltages depending on patient characteristics and CT systems. Differences in tube voltage affect Hounsfield Unit (HU) values measured in different tissues. However, so far, no study has actually studied the magnitude of the effect of the tube voltage on PCAT. Potential future cutoff values in PCAT need to be seen in perspective of difference by tube voltage, and may need adjustment by $\mathrm{kV}$ setting.

The objectives of this study were to explore PCAT $_{\mathrm{MA}}$ reference values of three main coronary arteries in patients without plaque on CCTA, and to determine the influence of cCTA tube voltages and vessel analyzed on $\mathrm{PCAT}_{\mathrm{MA}}$ measurement.

\section{Materials and methods}

\section{Study population}

This retrospective, single-center observational study was performed at the University Medical Center Groningen, Groningen, The Netherlands. The study was compliant with the Declaration of Helsinki. The study protocol was approved by the institutional ethical review board, and informed consent was waived. Patients were eligible if they were suspected of coronary artery disease and underwent routine cCTA between January 2015 and November 2017. The cohort list was randomly screened for patients meeting the inclusion criteria until the required sample size was reached; see sample size calculations in the statistical paragraph. Inclusion criteria were (1) calcium score of 0 and (2) no coronary plaque on cCTA. Exclusion criteria were (1) objection to the use of data for scientific research; (2) poor cCTA image quality; and (3) patients with anomalous coronary artery origin from the aorta sinus that leads to inaccurate measurements. A radiologist with 10 years of experience reevaluated all calcium scoring and cCTA scans. In case of doubt, a radiologist with 14 years of experience performed a second reading. 


\section{CCTA scan and post-processing protocol}

Third-generation dual-source CT was used (Somatom Force; Siemens Healthineers). A non-enhanced electrocardiography (ECG)-triggered $\mathrm{CT}$ acquisition was performed to obtain the calcium score. cCTA was performed according to standard clinical protocol. Patients received sublingual nitroglycerin unless contra-indicated. In case of high heart rate $(>70$ 73 beats $/ \mathrm{min}$ ), patients received an intravenous beta-blocker. cCTA was acquired in high-pitch mode in case of regular heart rate $<70$ beats $/ \mathrm{min}$, in sequential mode in diastolic phase if heart rate was $>70$ beats $/ \mathrm{min}$, and in sequential mode with broad ECG interval in case of arrhythmias. Tube voltage ranged from 70 to $120 \mathrm{kV}$, depending on patient size, as suggested by CarekV (kV optimization assistance). Contrast bolus timing was determined after a test bolus. Iomeprol (Iomeron 350; Bracco Altana Pharma) was injected with dose and flow rate depending on patient characteristics and scan mode. A dual bolus technique was used followed by a saline flush. cCTA images were reconstructed with a slice thickness of $0.6 \mathrm{~mm}$. Post-processing and analysis of cCTA images were performed using dedicated software (Aquarius iNtuition, TeraRecon, Version 4.4.13).

\section{PCAT $_{\text {MA }}$ measurement}

PCAT $_{M A}$ was measured in the main coronary arteries (LAD,

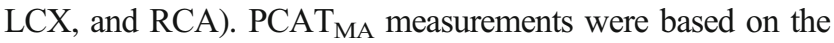
conceptual framework as proposed by Antonopoulos et al [16]. The workstation automatically reconstructed three-dimensional volume-rendered and curved multi-planar reformat images, which were manually corrected by the radiologist in case of identification errors. The following steps were performed (Fig. 1): (a) Start and end points of the PCAT $_{\mathrm{MA}}$ measurement were selected. For LAD and RCA, the start point was $10 \mathrm{~mm}$ distally from the origin to avoid overlap with the LCX measurement and influence of the aortic wall, respectively. For LCX, the vessel origin was selected as the start point. Because of LCX anatomy, there is limited adipose tissue around the LCX after $10 \mathrm{~mm}$. We adjusted the measurement length to $10 \mathrm{~mm}$ for all coronary arteries in order to minimize interference of side branch intersections, which costs less time than the $40 \mathrm{~mm}$ in the original study [16]. (b) A 1-mm gap was left around the artery lumen and the measurement circle in order to prevent blooming artifacts from high contrast concentration. (c) The mean HU value of adipose tissue was measured in a concentric circle from 1 to $2 \mathrm{~mm}$ around the coronary lumen (1 mm thickness). Compared with prior studies, we reduced measurement width from the average vessel diameter of about 3 to $1 \mathrm{~mm}$ in order to avoid interference from the myocardium and veins. (d) The software automatically calculates the mean $\mathrm{CT}$ attenuation and volume for voxels within the target threshold of -190 to $-30 \mathrm{HU}[16]$.

\section{EAT measurements}

The heart was manually segmented. Within this segmentation, an HU range from -190 to -30 was set to select relevant tissue. The volume value of the EAT was automatically obtained by the software.

\section{PCAT measurement methodology variation}

In order to evaluate whether measurement length influences PCAT $_{\mathrm{MA}}$ measurements, PCAT $\mathrm{MA}$ was measured with $40 \mathrm{~mm}$ and $10 \mathrm{~mm}$ lengths in 60 randomly selected cCTA scans. In twenty randomly selected cCTAs, intra- and interobserver agreements were determined. For intra-observer agreement, PCAT $_{\mathrm{MA}}$ was measured again by the same reader after at least 4 weeks to avoid image recognition. For interobserver agreement, a second independent reader measured $\mathrm{PCAT}_{\mathrm{MA}}$ after sufficient training.

\section{Statistical methods}

The sample size was calculated using paired sample $t$-testing with GPOWER software (Faul, Erdfelder, Lang, \& Buchner, version 3.1.9.2). For sample size calculation, we used results from two prior PCAT $_{\mathrm{MA}}$ studies [16, 25], with the following parameters: mean and SD of PCAT $_{\mathrm{MA}}(-75.1 \pm 8.6 \mathrm{HU}$ and $-77.0 \pm 8.5 \mathrm{HU})$; correlation between groups 0.5 . The effect size was calculated to be 0.2222108 . With $\alpha=0.05$, power $=$ 0.8 , and two-tailed analysis, the needed sample size was 161 . We added $20 \%$, yielding a total sample size of 192 , to decrease type I and type II error ratios.

Normality testing for continuous variables was performed with the Shapiro-Wilk test. Continuous variables were represented as mean $\pm \mathrm{SD}$. Categorical variables were recorded as numbers $(n)$ and frequencies (\%). Associations of age, sex, and BMI with PCAT $_{\mathrm{MA}}$ were tested using multivariable regression analysis. PCAT $_{\mathrm{MA}}$ values by sex were compared using unpaired sample $t$-testing. PCAT $_{\mathrm{MA}}$ comparisons between combinations of two coronary arteries were made using paired sample $t$-testing; values between three coronary arteries were compared using repeated ANOVA testing. In order to determine the effects of tube voltage on PCAT $\mathrm{TA}_{\mathrm{MA}}$, patients were grouped according to tube voltage as follows: 70, 80, $90,100,120 \mathrm{kV}$. For PCAT $\mathrm{MA}_{\mathrm{MA}}$ and EAT volume comparison of multiple $\mathrm{kV}$ groups, one-way ANOVA testing was used. Post hoc pairwise comparisons of PCAT $_{\mathrm{MA}}$ were performed between each two $\mathrm{kV}$ groups. $p$ values $<0.05$ were considered statistically significant. For multi-paired $t$-testing a Bonferroni correction was applied, adjusting the $p$ value accordingly. SPSS (SPSS, version 25; IBM) was used for statistical analysis. 
Fig. 1 Measurement steps of PCAT $_{\mathrm{MA}}$ (cCTA at $70 \mathrm{kV}$ in a 56year-old male patient). (a) Measurement ranges (red rectangles) are marked on the VR image, the $10-\mathrm{mm}$ reference line is the blue line. (b) A gap of $1 \mathrm{~mm}$ is determined around the border of the coronary lumen. (c) CT density is measured for a concentric ring from 1 to $2 \mathrm{~mm}$ around the coronary lumen $(1 \mathrm{~mm}$ thickness). (d) The software automatically calculates the mean $\mathrm{CT}$ attenuation and volume for voxels within the target threshold of -190 to $-30 \mathrm{HU}$. PCAT $_{\mathrm{MA}}$ is pericoronary adipose tissue mean attenuation; LAD is left anterior descending coronary artery; LCX is left circumflex coronary artery; $\mathrm{RCA}$ is right coronary artery; $\mathrm{VR}$ is volume rendering; cCTA is coronary computed tomography angiography

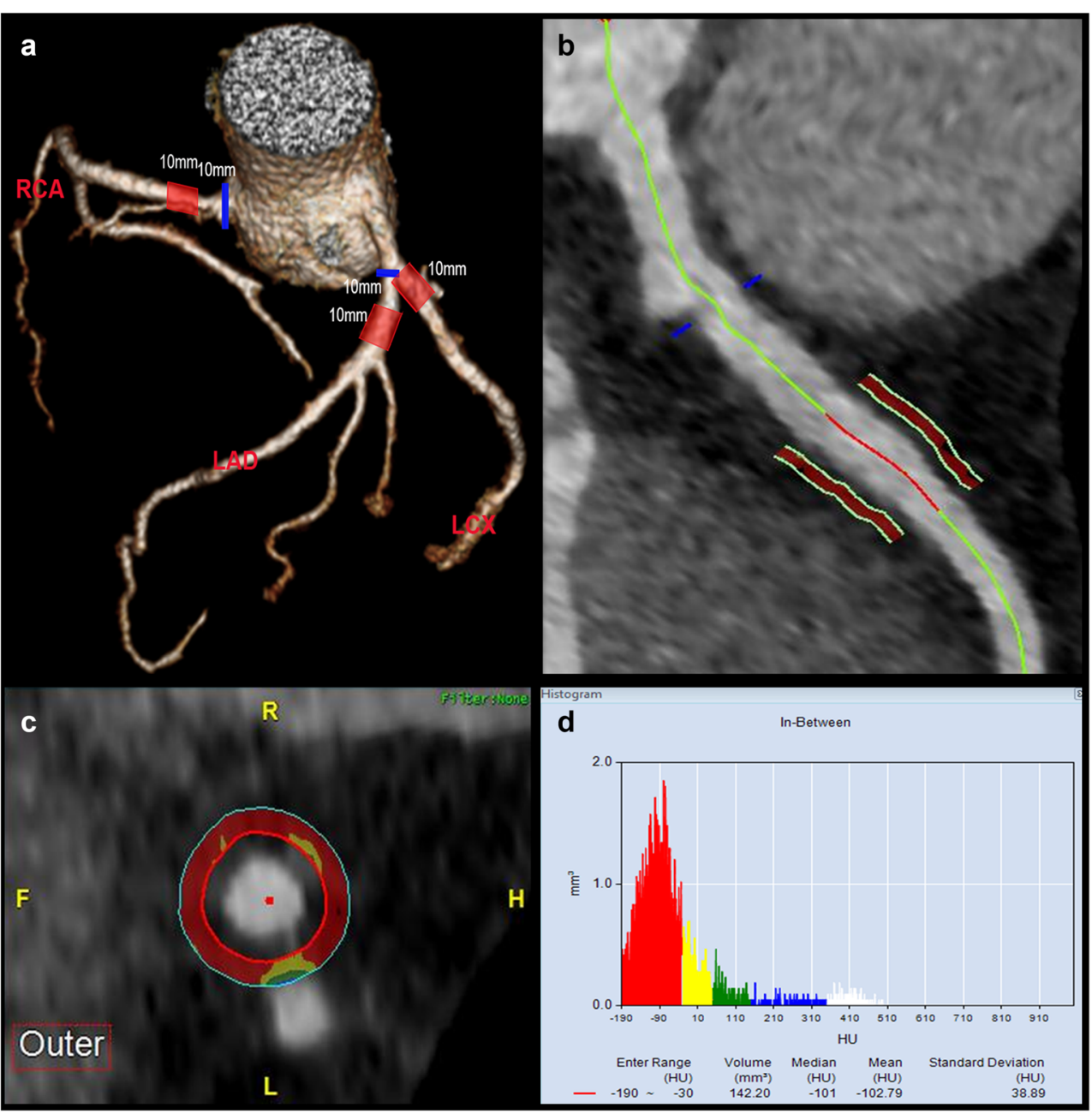

\section{Results}

\section{Study population characteristics}

In total, 206 patients without CAD on cCTA images were selected for analysis. Fourteen patients were excluded for various reasons: anomalous origin of coronary artery $(n=6)$, insufficient image quality $(n=5)$, incomplete coronary image coverage $(n=1)$, pacemaker artifact $(n=1)$, and streak artifact $(n=1)$ (Fig. 2). The final study population consisted of 192 patients (76 [39.6\%] men; mean age 50.5 years [range, 1979 years]) and 576 coronary arteries. Overall, 72 patients (37.5\%) underwent cCTA at $70 \mathrm{kV}, 53(27.6 \%)$ at $80 \mathrm{kV}$, $39(20.3 \%)$ at $90 \mathrm{kV}$, and $28(14.6 \%)$ at 100 to $120 \mathrm{kV}$ (Table 1).

\section{PCAT $_{\text {MA }}$ of healthy coronary arteries on CCTA}

Overall mean PCAT $\mathrm{TA}_{\mathrm{MA}}$ value was $-90.3 \pm 11.1 \mathrm{HU}$. Mean PCAT $_{\mathrm{MA}}$ of men and women was $-88.5 \pm 10.5 \mathrm{HU}$ and $91.5 \pm 11.3 \mathrm{HU}(p=0.001)$, respectively. In multivariable linear regression analysis, $\mathrm{kV}$, age, and gender were significantly associated with $\operatorname{PCAT}_{\mathrm{MA}}(p<0.05)$ while BMI was not $(p=0.235)$. Mean PCAT $_{\mathrm{MA}}$ of LAD, LCX, and RCA was $92.4 \pm 11.6 \mathrm{HU},-88.4 \pm 9.9 \mathrm{HU}$, and $-90.2 \pm 11.4 \mathrm{HU}$, respectively $(p<0.001)$. There were significant differences between all combinations of coronary arteries: $\mathrm{PCAT}_{\mathrm{MA}-\mathrm{LAD}}$ and PCAT $_{\text {MA-LCX }}(p<0.001)$, PCAT $_{\text {MA-LAD }}$ and PCAT MA- $_{\text {- }}$ RCA $(p=0.009), \operatorname{PCAT}_{\text {MA-LCX }}$ and $\operatorname{PCAT}_{\text {MA-RCA }}(p=0.033)$ (Fig. 3).

\section{Influence of tube voltage on $\mathrm{PCAT}_{\mathrm{MA}}$}

Mean PCAT $_{\mathrm{MA}}$ showed a positive linear association with tube voltage (Fig. 4). Mean (SD) $\mathrm{PCAT}_{\mathrm{MA}}$ of the $70 \mathrm{kV}$, $80 \mathrm{kV}, 90 \mathrm{kV}, 100 \mathrm{kV}$, and $120 \mathrm{kV}$ groups was $-95.6 \pm$ $9.6 \mathrm{HU},-90.2 \pm 11.5 \mathrm{HU},-87.3 \pm 9.9 \mathrm{HU},-82.7 \pm$ $6.2 \mathrm{HU}$, and $-79.3 \pm 6.8 \mathrm{HU}$, respectively $(p<0.001)$. Post hoc pairwise comparisons of the $\mathrm{kV}$ groups demonstrated significant differences between each two groups except for the $80 \mathrm{kV}$ and $90 \mathrm{kV}(p=0.222)$, and $100 \mathrm{kV}$ and $120 \mathrm{kV}$ groups $(p=0.267)$. 
Fig. 2 Flowchart of patient inclusion. CAD is coronary artery disease; cCTA is coronary computed tomography angiography

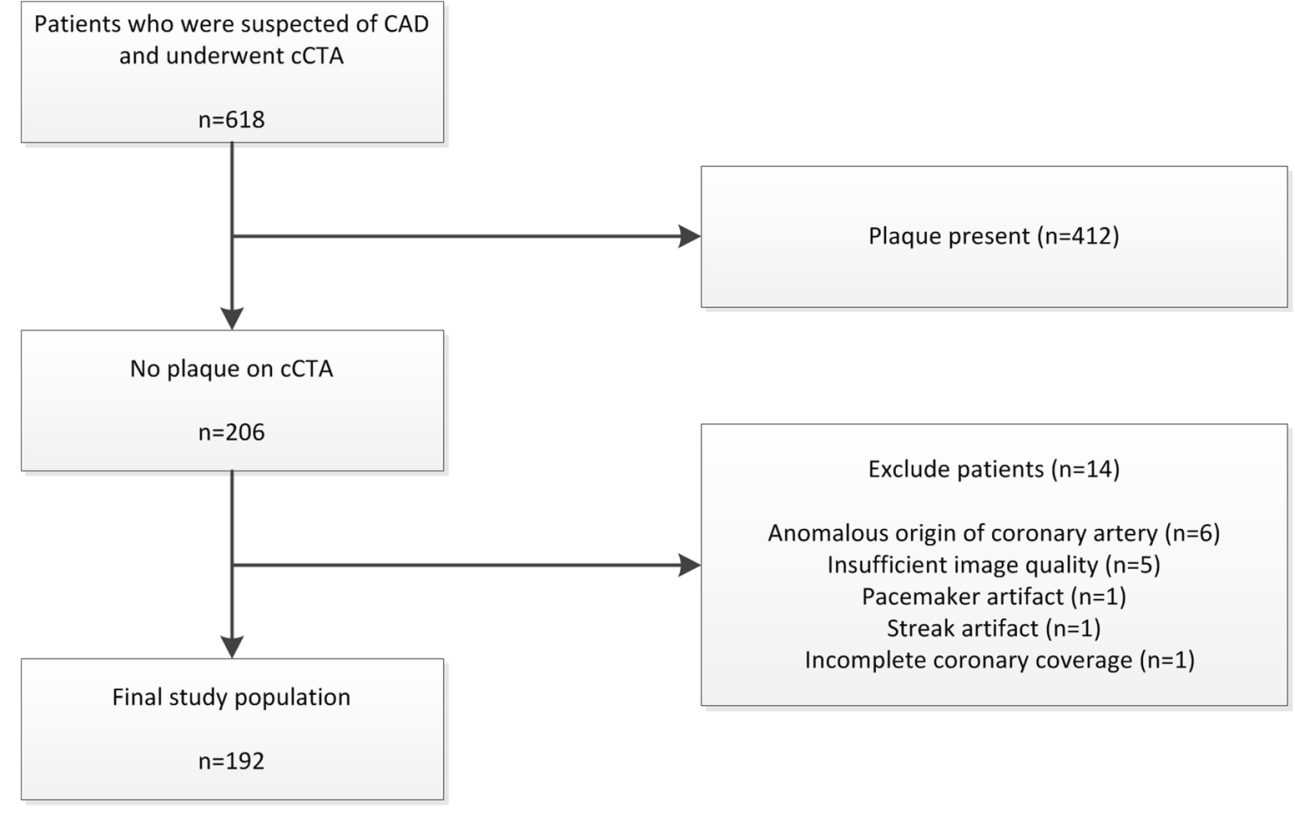

\section{Tube voltage and EAT volume}

Mean EAT volume showed a positive linear association with tube voltage (Fig. 5). Mean (SD) EAT volume of the $70 \mathrm{kV}$, $80 \mathrm{kV}, 90 \mathrm{kV}, 100 \mathrm{kV}$, and $120 \mathrm{kV}$ groups was $107.6 \pm$

Table 1 Patients' baseline characteristics. Body mass index information was available for 108 patients. $k V$ is tube voltage, $S D$ is standard deviation, $c C T A$ is coronary computed tomography angiography

\begin{tabular}{ll}
\hline Variables & Overall $(n=192)$ \\
\hline Age, years, mean \pm SD & $50.5 \pm 11.5$ \\
Men, $n(\%)$ & $76(39.6 \%)$ \\
Body mass index, mean $\pm \mathrm{SD}$ & $26.4 \pm 5.0$ \\
Risk factor, $n(\%)$ & \\
Diabetes mellitus & $13(6.8 \%)$ \\
Hypertension & $70(36.5 \%)$ \\
Hyperlipidemia & $34(17.7 \%)$ \\
Former smoker & $38(19.8 \%)$ \\
Current smoker & $37(19.3 \%)$ \\
Family history of coronary artery disease & $68(35.4 \%)$ \\
Indication for cCTA, $n(\%)$ & \\
Typical angina & $15(7.8 \%)$ \\
Atypical angina & $100(52.1 \%)$ \\
Non-anginal chest pain & $14(7.3 \%)$ \\
Dyspnea/dyspnea' effort & $12(6.3 \%)$ \\
Other & $51(26.6 \%)$ \\
Tube voltage, $n(\%)$ & $72(37.5 \%)$ \\
$70 \mathrm{kV}$ & $53(27.6 \%)$ \\
$80 \mathrm{kV}$ & $39(20.3 \%)$ \\
$90 \mathrm{kV}$ & $28(14.6 \%)$ \\
$100-120 \mathrm{kV}$ &
\end{tabular}

$49.7 \mathrm{~cm}^{3}, 145.5 \pm 60.1 \mathrm{~cm}^{3}, 172.8 \pm 63.6 \mathrm{~cm}^{3}, 183.7 \pm$ $63.2 \mathrm{~cm}^{3}$, and $199.5 \pm 78.1 \mathrm{~cm}^{3}$, respectively $(p<0.001)$.

\section{PCAT measurement methodology variation}

PCAT $_{\mathrm{MA}}$ of LAD, LCX, and RCA for 40-mm measurement length was $-95.6 \pm 9.7 \mathrm{HU},-88.7 \pm 10.0 \mathrm{HU}$, and $-92.9 \pm$

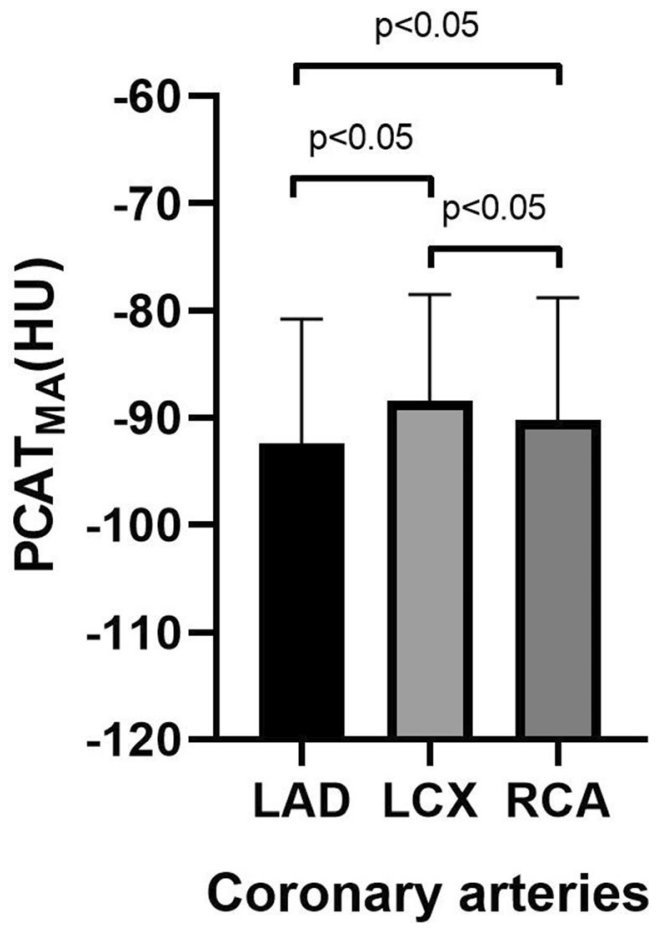

Fig. 3 PCAT $_{\mathrm{MA}}$ values for the main coronary arteries. PCAT $_{\mathrm{MA}}$ is pericoronary adipose tissue mean attenuation; LAD is left anterior descending coronary artery; LCX is left circumflex coronary artery; $\mathrm{RCA}$ is right coronary artery 
PCAT $_{M A}$

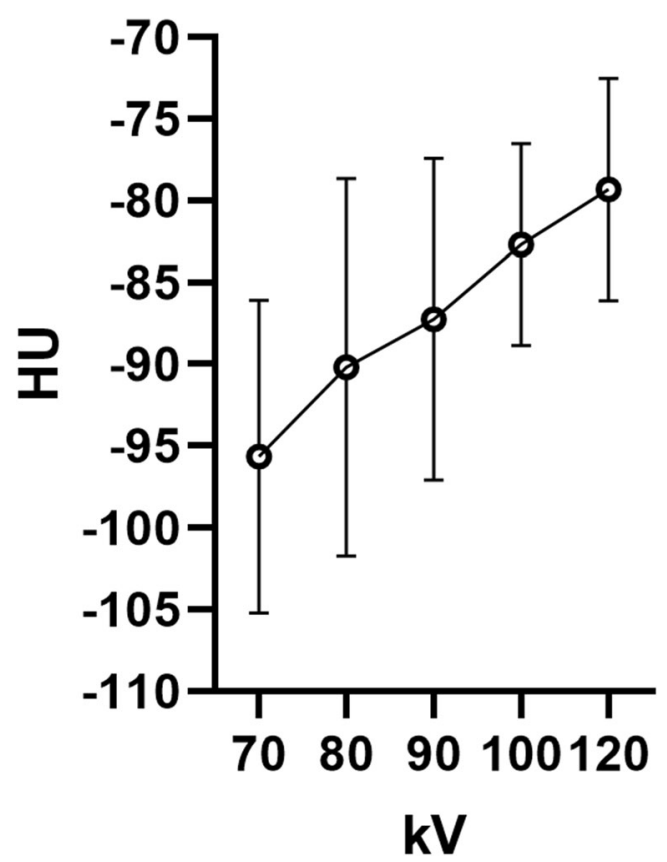

Fig. 4 PCAT $_{\mathrm{MA}}$ values in patient groups based on cCTA tube voltage setting. PCAT $_{M A}$ is pericoronary adipose tissue mean attenuation; $\mathrm{kV}$ is kilovoltage; HU is Hounsfield Units; cCTA is coronary computed tomography angiography

8.3 HU, respectively, compared with $-94.5 \pm 11.0 \mathrm{HU},-$ $90.0 \pm 8.7 \mathrm{HU}$, and $-91.6 \pm 9.7 \mathrm{HU}$ for $10-\mathrm{mm}$ measurement length ( $p=0.124,0.118,0.116$, respectively) (Supplementary Material Table S1 and Fig. S1). There was excellent correlation within and between readers for repeated $\mathrm{PCAT}_{\mathrm{MA}}$

\section{EAT Volume}

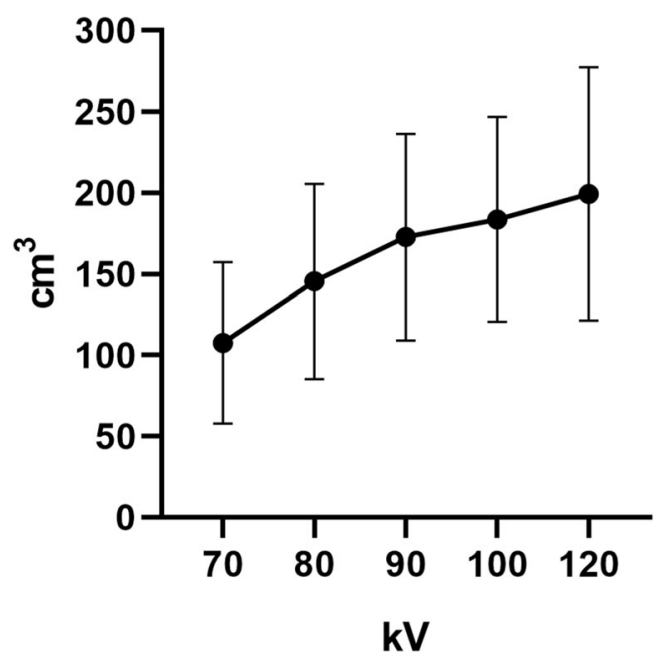

Fig. 5 EAT volume in patient groups based on cCTA tube voltage setting. EAT is epicardial adipose tissue; $\mathrm{kV}$ is kilovoltage; $\mathrm{CM}$ is centimeter; cCTA is coronary computed tomography angiography measurements (0.974-0.982), with minimal bias but with some variability between readings (upper and lower limits of agreement 3.9 HU and - 3.3 HU within-reader, 4.6 HU, and 4.1 HU between-readers) (Supplementary Material Table S2 and Fig. S2).

\section{Discussion}

In this study, we investigated the $\mathrm{PCAT}_{\mathrm{MA}}$ values of healthy coronary arteries and the influence of tube voltage. Our main results showed that the tube voltage of CCTA significantly influenced PCAT $_{M A}$ values in patients without $C A D$, and that PCAT $_{\mathrm{MA}}$ differed slightly between the LAD, LCX, and RCA.

Although the presence of obstructive disease on cCTA is associated with worse outcomes, many myocardial infarctions originate from coronary segments without prior obstructive disease. Thus, the focus has shifted to the identification of segments at future risk of developing potentially vulnerable plaque [26]. Studies on PCAT $_{M A}$ in diseased populations showed significant differences between diseased and nondiseased coronary arteries, and between flow-limiting and non-flow limiting stenosis [24]. Although results were statistically significant, these studies show, similar to the current study, limited absolute differences in PCAT values $( \pm 5 \mathrm{HU})$. However, these studies show an increased accuracy for the prediction of hemodynamic significance of a lesion, especially in combination with other factors such as stenosis diameter. Further understanding of the PCAT parameter and the influence of scan protocol settings on this biomarker and its variability can help to determine limits of reliability around PCAT $_{M A}$ values when comparing patients.

One of the main results of this study is that the use of different $\mathrm{kV}$ levels has considerable impact on $\mathrm{PCAT}_{\mathrm{MA}}$ values. In clinical practice, cCTA acquisitions are acquired with varying $\mathrm{kV}$ levels. Higher $\mathrm{kV}$ voltages will inherently lead to higher PCAT $_{\mathrm{MA}}$ and EAT attenuation, unrelated to a pathophysiological process. Prior studies investigating the use of PCAT $_{\mathrm{MA}}$ used cCTA images obtained at $100 \mathrm{kV}$ and/or $120 \mathrm{kV}[16,20,21,25]$. However, lower $\mathrm{kV}$ acquisitions are becoming increasingly popular in order to reduce radiation and contrast medium volume. Recent results from the PROTECTION VI Study showed that low kV settings for cCTA $(<100 \mathrm{kV})$ are already applied in $14 \%$ of patients and this is only expected to increase [27, 28]. Our results showed significant differences between all $\mathrm{kV}$ levels except between 80 and $90 \mathrm{kV}$, and between 100 and $120 \mathrm{kV}$. The lack of difference in PCAT $_{\mathrm{MA}}$ between 100 and $120 \mathrm{kV}$ is also reflected by the similar PCAT $_{\mathrm{MA}}$ results from previous studies investigating only those two levels. The differences in $\mathrm{PCAT}_{\mathrm{MA}}$ between the other $\mathrm{kV}$ levels indicate that a $\mathrm{kV}$ specific PCAT $_{\mathrm{MA}}$ cutoff should be used to discriminate healthy from diseased patients and perform accurate risk 
assessment. Determination of this cutoff fell outside the scope of this research. The current study provides reference values of PCAT $_{\mathrm{MA}}$ by $\mathrm{kV}$ setting in normal coronary arteries; future studies should investigate the $\mathrm{PCAT}_{\mathrm{MA}}$ by $\mathrm{kV}$ in coronary arteries with plaque and/or stenosis, and evaluate the optimal cutoff values. To construct a $\mathrm{kV}$ correction factor, ideally the same patients would have to undergo repeated cCTA at different $\mathrm{kV}$ levels. The results also showed a positive relation between $\mathrm{kV}$ and EAT volume. This is likely mostly due to the fact that patients with higher BMI (and more intrathoracic fat) were usually scanned with higher $\mathrm{kV}$ to get better image quality. In view of the influence of $\mathrm{kV}$ on PCAT $_{\mathrm{MA}}$ analysis, it is recommended that for the longitudinal follow-up and compar-

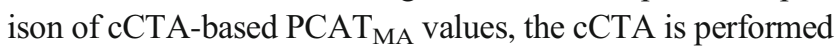
at equal tube voltage setting.

The majority of PCAT $_{\mathrm{MA}}$ studies about relationship with CAD focused on analysis of the RCA alone, while the LAD and LCX could provide additional information and increase the accuracy of outcome prediction $[16,21]$. Our study focused on PCAT $_{\mathrm{MA}}$ measurements in all three coronary arteries, showing that $\mathrm{PCAT}_{\mathrm{MA}}$ was slightly but significantly different between the LAD, LCX, and RCA. This difference could be caused by differences in anatomy and surrounding tissues, indicating that $\mathrm{PCAT}_{\mathrm{MA}}$ values and corresponding cutoff values based on RCA measurements cannot be directly transferred to the other coronary arteries. Our study results showed that LAD had slightly but significantly lower PCAT $_{\mathrm{MA}}$ compared with the RCA and LCX. As is known in literature, atherosclerosis development also differs between the coronary arteries. The LAD is subject to atherosclerosis more often and at an earlier stage in comparison with RCA and LCX [29-31]. The fact that LAD had a lower PCAT ${ }_{\mathrm{MA}}$ is an important hypothesis-generating finding. This finding suggests that PCAT $_{\mathrm{MA}}$ could be related to vessel vulnerability for atherosclerosis. This hypothesis should be further investigated in pathophysiological and prospective clinical studies.

To analyze all three coronary arteries, an adjusted measurement method was used in our study. Previously, a measurement length of $40 \mathrm{~mm}$ was used $[16,21,25]$ which was feasible for the RCA because it has fewer side branches and proximal variations than the LAD and LCX. To avoid influence of side branches, measurement length was reduced from 40 to $10 \mathrm{~mm}$ in this study. Results from our sub-study demonstrated no differences in PCAT $\mathrm{MA}_{\mathrm{MA}}$ between our 10-mm methods and $40-\mathrm{mm}$ method. PCAT $\mathrm{MA}$ measurement width around the coronary could potentially affect measurement accuracy. Prior studies measured PCAT $\mathrm{MA}_{\mathrm{MA}}$ using approximately $3 \mathrm{~mm}$ thickness (or equal to vessel diameter) around the coronary vessels $[16,20,21,25]$. However, contrast enhancement of the lumen has been found to influence the HU values in the voxels adjacent to the luminal border [32]. To take this into account, we applied a 1-mm gap around the vessel wall. Thus, our study measured PCAT $_{\mathrm{MA}}$ using a more constrictive measurement width, making it more suitable for LAD and LCX measurements, and potentially more sensitive to inflammatory changes. Manual PCAT measurements using the method described here can be performed in a similar time span compared with manual EAT measurements. Fully automated software, as mentioned by some researchers [21], allows for PCAT $_{M A}$ evaluation within $30 \mathrm{~s}$, increasing the time efficiency of PCAT $_{M A}$ analysis and enabling use in clinical practice.

While PCAT $_{\mathrm{MA}}$ and EAT both are measures of adipose tissue, they represent different processes [16]. PCAT $_{\mathrm{MA}}$ quantifies fat at the per-vessel level or per-lesion level as an indicator of coronary inflammation, while EAT provides a measure of the volume of the entire epicardial fat system as a marker for paracrine effects of fat. Thus, PCAT $_{\mathrm{MA}}$ may provide a more specific, focal assessment of coronary risk and vulnerability. $\mathrm{PCAT}_{\mathrm{MA}}$ was shown to have additional diagnostic value with more precision and specificity compared with EAT measurements [33]. Studies found that PCAT $_{\mathrm{MA}}$ and FFR were related at the per-vessel level [20] and that PCAT $_{\mathrm{MA}}$ of RCA was able to assist in risk stratification of cardiovascular mortality [25]. The combined use of PCAT $_{\mathrm{MA}}$, total plaque volume, and diameter stenosis has shown high diagnostic accuracy for prediction of hemodynamically significant coronary stenosis [24]. Interestingly, Goeller et al [21] found that changes in attenuation of adipose tissue in the pericoronary space were related to changes in plaque burden. These results suggest that these effects are associated with changes in PCAT specifically rather than adipose tissue in general.

Besides differences between $\mathrm{kV}$ levels and coronary arteries, our results show a slight but important difference in PCAT $_{M A}$ between men and women that could not be explained by differences in $\mathrm{kV}$ distribution. Men are known to get CAD more frequently than women and at an earlier age [31]. The PCAT $\mathrm{MA}_{\mathrm{MA}}$ difference between men and women follows the same trend. This finding deserves further exploration in a study with comprehensive cardiovascular risk factor assessment and more diverse range of coronary atherosclerosis.

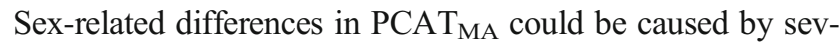
eral factors. Men have a higher amount of EAT than women $[34,35]$, related to cardiovascular risk. There are sex-related differences in the regulation mechanism of pericardial adipokines [36] and in the physiological mechanism of adipose tissue [37]. Additionally, it could be that differences in sex-related hormones, higher low-density lipoprotein in male patients, and differences in risk factors impact PCAT $_{\mathrm{MA}}$ in men and women [38].

\section{Limitations}

This was a single-center retrospective study of patients with a clinical indication for cCTA. There was no follow-up, since a normal cCTA result led to discharge from the cardiology outpatient clinic. Some of the patients might have been at higher 
risk to develop CAD, reflected in already altered PCAT $_{M A}$ values while there was no plaque development yet. There could be factors other than tube voltage affecting PCAT $_{\mathrm{MA}}$ measurements such as obesity or diabetes, inflammatory processes, or specific medication. Further work is needed to explore all potentially influencing factors. With regard to the measurement itself, the anatomical variation of LCX compared with LAD and RCA was relatively large and could have affected the PCAT $_{\mathrm{MA}}$ measurement. However, with our adjusted measurement protocol, we found excellent correlation between repeated measurements within and between readers with limited bias, indicating the validity of the PCAT $\mathrm{MA}$ measurement.

\section{Conclusion}

In conclusion, our results showed that $\mathrm{PCAT}_{\mathrm{MA}}$ varied considerably by tube voltage in patients without plaque on cCTA, with minor differences in PCAT $_{\mathrm{MA}}$ between coronary arteries (LAD, LCX, RCA). cCTA kV setting needs to be taken into account when interpreting PCAT $_{\mathrm{MA}}$ values.

Acknowledgements We thank Dr. Estelle Noach for critical reading of the manuscript and Mrs. Amanda Boone for technical support of the Aquarius software. The UMCG is supported by an institutional research grant from Siemens Healthineers. We are grateful to Dr. Mieneke Rook for the scientific advice at the beginning of this study.

Funding information Financial support provided by the China Scholarship Council (CSC) for the $\mathrm{PhD}$ project of the first author is gratefully acknowledged.

\section{Compliance with ethical standards}

Guarantor The scientific guarantor of this publication is Rozemarijn Vliegenthart.

Conflict of interest The UMCG is supported by an institutional research grant from Siemens Healthineers. The authors of this manuscript declare no other relationships with any companies whose products or services may be related to the subject matter of the article.

Statistics and biometry One of the authors has significant statistical expertise.

Informed consent Written informed consent was waived by the Institutional Review Board.

Ethical approval Institutional Review Board approval was obtained.

Methodology
- retrospective
- cross-sectional study
- performed at one institution

Open Access This article is licensed under a Creative Commons Attribution 4.0 International License, which permits use, sharing, adaptation, distribution and reproduction in any medium or format, as long as you give appropriate credit to the original author(s) and the source, provide a link to the Creative Commons licence, and indicate if changes were made. The images or other third party material in this article are included in the article's Creative Commons licence, unless indicated otherwise in a credit line to the material. If material is not included in the article's Creative Commons licence and your intended use is not permitted by statutory regulation or exceeds the permitted use, you will need to obtain permission directly from the copyright holder. To view a copy of this licence, visit http://creativecommons.org/licenses/by/4.0/.

\section{References}

1. Stoll G, Bendszus M (2006) Inflammation and atherosclerosis: novel insights into plaque formation and destabilization. Stroke 37: 1923-1932

2. Hansson GK (2005) Inflammation, atherosclerosis, and coronary artery disease. N Engl J Med 352:1685-1695

3. Libby P, Tabas I, Fredman G, Fisher EA (2014) Inflammation and its resolution as determinants of acute coronary syndromes. Circ Res 114:1867-1879

4. Choi BJ, Matsuo Y, Aoki T et al (2014) Coronary endothelial dysfunction is associated with inflammation and vasa vasorum proliferation in patients with early atherosclerosis. Arterioscler Thromb Vasc Biol 34:2473-2477

5. Ridker PM, Libby P, MacFadyen JG et al (2018) Modulation of the interleukin-6 signalling pathway and incidence rates of atherosclerotic events and all-cause mortality: analyses from the Canakinumab Anti-Inflammatory Thrombosis Outcomes Study (CANTOS). Eur Heart J 39:3499-3507

6. Ridker PM, Everett BM, Thuren T et al (2017) Antiinflammatory therapy with canakinumab for atherosclerotic disease. N Engl J Med 377:1119-1131

7. Nosalski R, Guzik TJ (2017) Perivascular adipose tissue inflammation in vascular disease. Br J Pharmacol 174:3496-3513

8. Mancio J, Oikonomou EK, Antoniades C (2018) Perivascular adipose tissue and coronary atherosclerosis. Heart 104:1654-1662

9. Tanaka K, Sata M (2018) Roles of perivascular adipose tissue in the pathogenesis of atherosclerosis. Front Physiol 9:3

10. Ohyama K, Matsumoto Y, Takanami K et al (2018) Coronary adventitial and perivascular adipose tissue inflammation in patients with vasospastic angina. J Am Coll Cardiol 71:414-425

11. Mazurek T, Zhang L, Zalewski A et al (2003) Human epicardial adipose tissue is a source of inflammatory mediators. Circulation 108:2460-2466

12. Goeller M, Achenbach S, Marwan M et al (2018) Epicardial adipose tissue density and volume are related to subclinical atherosclerosis, inflammation and major adverse cardiac events in asymptomatic subjects. J Cardiovasc Comput Tomogr 12:67-73

13. Lu MT, Park J, Ghemigian K et al (2016) Epicardial and paracardial adipose tissue volume and attenuation - association with high-risk coronary plaque on computed tomographic angiography in the ROMICAT II trial. Atherosclerosis 251:47-54

14. Nagayama Y, Nakamura N, Itatani R et al (2019) Epicardial fat volume measured on nongated chest $\mathrm{CT}$ is a predictor of coronary artery disease. Eur Radiol 29:3638-3646

15. Bos D, Leening MJG (2018) Leveraging the coronary calcium scan beyond the coronary calcium score. Eur Radiol 28:3082-3087

16. Antonopoulos AS, Sanna F, Sabharwal N et al (2017) Detecting human coronary inflammation by imaging perivascular fat. Sci Transl Med 9:eaal2658 
17. Lian X, Gollasch M (2016) A clinical perspective: contribution of dysfunctional perivascular adipose tissue (PVAT) to cardiovascular risk. Curr Hypertens Rep 18:82

18. Margaritis M, Antonopoulos AS, Digby J et al (2013) Interactions between vascular wall and perivascular adipose tissue reveal novel roles for adiponectin in the regulation of endothelial nitric oxide synthase function in human vessels. Circulation 127:2209-2221

19. Antoniades C, Antonopoulos AS, Tousoulis D, Stefanadis C (2009) Adiponectin: from obesity to cardiovascular disease. Obes Rev 10: 269-279

20. Goeller M, Achenbach S, Cadet S et al (2018) Pericoronary adipose tissue computed tomography attenuation and high-risk plaque characteristics in acute coronary syndrome compared with stable coronary artery disease. JAMA Cardiol 3:858-863

21. Goeller M, Tamarappoo BK, Kwan AC et al (2019) Relationship between changes in pericoronary adipose tissue attenuation and coronary plaque burden quantified from coronary computed tomography angiography. Eur Heart J Cardiovasc Imaging 20:636-643

22. Kwiecinski J, Dey D, Cadet S et al (2019) Peri-coronary adipose tissue density is associated with (18)F-sodium fluoride coronary uptake in stable patients with high-risk plaques. JACC Cardiovasc Imaging 12:2000-2010

23. Gaibazzi N, Martini C, Botti A, Pinazzi A, Bottazzi B, Palumbo AA (2019) Coronary inflammation by computed tomography pericoronary fat attenuation in MINOCA and Tako-Tsubo syndrome. J Am Heart Assoc 8:e013235

24. Yu M, Dai X, Deng J, Lu Z, Shen C, Zhang J (2020) Diagnostic performance of perivascular fat attenuation index to predict hemodynamic significance of coronary stenosis: a preliminary coronary computed tomography angiography study. Eur Radiol 30:673-681

25. Oikonomou EK, Marwan M, Desai MY et al (2018) Non-invasive detection of coronary inflammation using computed tomography and prediction of residual cardiovascular risk (the CRISP CT study): a post-hoc analysis of prospective outcome data. Lancet 392:929-939

26. Maddox TM, Stanislawski MA, Grunwald GK et al (2014) Nonobstructive coronary artery disease and risk of myocardial infarction. JAMA 312:1754-1763

27. Stocker TJ, Deseive S, Leipsic J et al (2018) Reduction in radiation exposure in cardiovascular computed tomography imaging: results from the PROspective multicenter registry on radiaTion dose Estimates of cardiac CT angIOgraphy iN daily practice in 2017 (PROTECTION VI). Eur Heart J 39:3715-3723
28. Stocker TJ, Leipsic J, Hadamitzky M et al (2020) Application of low tube potentials in CCTA: results from the PROTECTION VI study. JACC Cardiovasc Imaging 13:425-434

29. Alluri K, McEvoy JW, Dardari ZA et al (2015) Distribution and burden of newly detected coronary artery calcium: results from the multi-ethnic study of atherosclerosis. J Cardiovasc Comput Tomogr 9:337-344 e331

30. Wykrzykowska JJ, Mintz GS, Garcia-Garcia HM et al (2012) Longitudinal distribution of plaque burden and necrotic core-rich plaques in noncuprit lesions of patients presenting with acute coronary syndromes. JACC Cardiovasc Imaging 5:S10-S18

31. Schulman-Marcus J, Hartaigh B, Gransar H et al (2016) Sexspecific associations between coronary artery plaque extent and risk of major adverse cardiovascular events: the CONFIRM long-term registry. JACC Cardiovasc Imaging 9:364-372

32. Kristanto W, Tuncay V, Vliegenthart R, van Ooijen PM, Oudkerk M (2015) Correction of lumen contrast-enhancement influence on non-calcified coronary atherosclerotic plaque quantification on CT. Int J Cardiovasc Imaging 31:429-436

33. Maurovich-Horvat P, Kallianos K, Engel LC et al (2015) Relationship of thoracic fat depots with coronary atherosclerosis and circulating inflammatory biomarkers. Obesity (Silver Spring) 23:1178-1184

34. Gill CM, Azevedo DC, Oliveira AL, Martinez-Salazar EL, Torriani M, Bredella MA (2018) Sex differences in pericardial adipose tissue assessed by PET/CT and association with cardiometabolic risk. Acta Radiol 59:1203-1209

35. Mancio J, Pinheiro M, Ferreira W et al (2017) Gender differences in the association of epicardial adipose tissue and coronary artery calcification: EPICHEART study: EAT and coronary calcification by gender. Int J Cardiol 249:419-425

36. Fei J, Cook C, Blough E, Santanam N (2010) Age and sex mediated changes in epicardial fat adipokines. Atherosclerosis 212:488-494

37. Chang E, Varghese M, Singer K (2018) Gender and sex differences in adipose tissue. Curr Diab Rep 18:69

38. Arnold AP, Cassis LA, Eghbali M, Reue K, Sandberg K (2017) Sex hormones and sex chromosomes cause sex differences in the development of cardiovascular diseases. Arterioscler Thromb Vasc Biol $37: 746-756$

Publisher's note Springer Nature remains neutral with regard to jurisdictional claims in published maps and institutional affiliations. 